\title{
FIP1L1-PDGFRA fusion-negative hypereosinophilic syndrome with uncommon cardiac involvement responding to imatinib treatment: A case report
}

\author{
AMANDA SANTOS DAL BERTO ${ }^{1}$, RICARDO HOHMANN CAMIÑA ${ }^{1}$, \\ EDUARDO SILVA MACHADO ${ }^{1-3}$ and ANTUANI RAFAEL BAPTISTELLA ${ }^{1,2,4,5}$
}

\author{
${ }^{1}$ Santa Terezinha University Hospital; ${ }^{2}$ University of West Santa Catarina; ${ }^{3}$ Department of Clinical Oncology, \\ Santa Terezinha University Hospital; ${ }^{4}$ Oncology Research Group of Santa Terezinha University \\ Hospital /University of West Santa Catarina; ${ }^{5}$ Post Graduation Program in Bioscience and \\ Health/University of West Santa Catarina, Joaçaba, Santa Catarina 89600-000, Brazil
}

Received April 17, 2018; Accepted May 21, 2018

DOI: $10.3892 / \mathrm{mco} .2018 .1637$

\begin{abstract}
Hypereosinophilic syndrome is a rare, chronic hematological disease characterized by a persistently elevated eosinophil count exceeding $1.5 \times 10^{9} / 1$, following the exclusion of other potential etiologies. The systemic involvement of the disease causes tissue damage through eosinophil infiltration, and may affect various organs; cardiac complications are observed in 50-60\% of cases, which are predominately attributed to endomyocardial fibrosis. The treatment is based initially on determining the presence of the FIP1L1-PDGFRA fusion. Patients with positive results for this mutation tend to achieve a complete response with imatinib treatment, which is thus the first line of treatment for this condition. However, patients who are negative for this mutation initially undergo treatment with corticosteroids. This study reports the case of a male 53-year-old patient diagnosed with hypereosinophilic syndrome in 2012, with negative results for the FIP1L1-PDGFRA mutation, and persistently high eosinophil levels, despite receiving the second line of standard treatment for this condition with hydroxyurea, and having already used corticosteroids without success. At the time of admission, the patient presented with acute decompensated heart failure due to severe mitral regurgitation, without any evidence of prior myocardial fibrosis or restrictive cardiomyopathy, and without suggestion of an associated ventricular hypertrophy. This clinical presentation is uncommon, as valvular involvement usually appears in the third stage of the development of cardiac involvement, and is usually associated with fibrosis and thrombotic events. Alternative
\end{abstract}

Correspondence to: Professor Antuani Rafael Baptistella, Santa Terezinha University Hospital, Travessa Domingos Bonato, 37-CEP, Joaçaba, Santa Catarina 89600-000, Brazil

E-mail: antuani.baptistella@unoesc.edu.br

Key words: hypereosinophilic syndrome, severe mitral regurgitation, imatinib, negative FIPIL1-PDGFRA fusion therapeutic possibilities were evaluated due to the significant progression of the disease, and it was decided to attempt the use of imatinib, despite its use being preferably recommended for FIPIL1-PDGFRA-positive patients. The patient exhibited an evident and immediate response to imatinib, with normalization of the eosinophil count within $24 \mathrm{~h}$ of the first dose, which was maintained for at least the next 19 months. This clinical presentation is uncommon, as patients negative for FIPIL1-PDGFRA fusion do not frequently respond to imatinib treatment, and symptomatic heart failure usually appears in the third stage of disease progression.

\section{Introduction}

Hypereosinophilic syndrome is a rare hematological condition characterized by the overproduction of eosinophils $\left(>1.5 \times 10^{9} / 1\right)$ by the bone marrow in an idiopathic manner, which is associated with tissue infiltration and lesions to multiple organs, with a duration of $>6$ months, or on two separate occasions, excluding other causes of increased eosinophil count, including medication, allergies, viral infection, parasitic diseases or cancer, among others (1-3). The term 'hypereosinophilic syndrome' was introduced in 1968 by Hardy and Anderson after the evaluation of three clinical cases associated with the production and maintenance of high levels of eosinophils without other apparent causes (4). Since then, case reports with descriptions of the complications have been published in the literature, although it is not yet possible to accurately estimate the prevalence and incidence of the disease (5).

According to new criteria, signs or symptoms of organ involvement are not required for diagnosis, since the patients may be initially asymptomatic and develop symptoms of organ involvement as the disease progresses (6).

The mechanism underlying eosinophilic hyperproliferation remains unknown, but the pathophysiology is dependent on the deregulation of the production of eosinophils (7). Activated eosinophils may infiltrate various tissues, releasing a wide variety of mediators inducing damage or dysfunction (3). New studies are being conducted, and remarkable progress has been 
achieved, including the identification of the FIP1L1-PDGFRA fusion, the most frequent mutation found in hypereosinophilic syndrome $(1,6)$.

A distinction should be made between the disorder characterized by a local increase in eosinophils without a progressive increase of eosinophils in the peripheral blood, with deposition in a single organ, as is the case in eosinophilic asthma or eosinophilic dermatitis, and the hypereosinophilic syndrome, which may affect one isolated organ or several systems, as the production of eosinophils by the bone marrow is deregulated (5). The systemic involvement of this disease occurs through the activation of eosinophils, a tendency for thrombotic events, the release of the eosinophil granule contents and protein deposition $(3,8,9)$.

Cardiac involvement associated with hypereosinophilic syndrome is frequent, observed in $\sim 50-60 \%$ of the cases (10). In 1936, Loeffler first described the association between eosinophilia and active carditis, with the latter becoming known as Loeffler endocarditis, which is characterized by eosinophilic myocarditis with a tendency for endomyocardial fibrosis. Loeffler endocarditis, or eosinophilic endocardial disease, or endomyocardial fibrosis, is the predominant form of cardiac involvement. The damage to the endocardium and myocardium is caused by toxic effects associated with the degradation of eosinophils, and defines tissue infiltration as a precipitating factor of local inflammation and subsequent fibrosis (10).

In hypereosinophilic syndrome, treatment is not always initiated at the time of diagnosis, with patients evaluated on an individual basis in accordance with the characterization and eosinophil count, the evidence of systemic involvement and/or the rate of disease progression $(3,5)$.

Therapeutic decisions are mainly based on the presence of the FIP1L1-PDGFRA fusion. Patients who test positive for FIP1L1-PDGFRA respond to treatment with tyrosine kinase inhibitors, such as imatinib $(4,5)$, whereas patients who test negative for FIP1L1-PDGFRA should initially receive treatment with corticosteroids, with the dose depending on the clinical symptoms and laboratory findings. As a second choice, the use of hydroxyurea is recommended for these patients, and initial therapy combined with the use of corticosteroids may also be recommended, with its efficacy depending on the successful inhibition of eosinophil production $(5,11)$.

The first line of treatment for patients without the FIP1L1-PDGFRA mutation includes the administration of corticosteroids (5), whereas when the mutation is present, the use of imatinib is the first choice of treatment $(4,5)$. Cardiac involvement is frequent, and is most commonly observed at advanced stages of endomyocardial fibrosis (10). To the best of our knowledge, this is the first case of a complete and sustained response to imatinib in a patient negative for FIP1L1-PDGFRA, who had also developed heart failure with development of isolated mitral regurgitation, without evidence of associated restrictive heart disease and/or evidence of associated left ventricular hypertrophy.

\section{Case report}

A 53-year-old male patient, resident in the Middle West region of Santa Catarina, Brazil, presented at the Emergency Department of the Hospital Universitário Santa Terezinha (HUST) in December 2015 with complaints of dyspnea associated with paroxysmal nocturnal dyspnea and fatigue on moderate and mild exertion, starting 2 months earlier and progressively worsening. When describing medical history, the patient reported suffering from diagnosed hypereosinophilic syndrome and receiving treatment with hydroxyurea (2,500 mg/day) for $\sim 6$ months; he refuted the presence of other comorbidities or the continuous use of other medication. The patient was followed up by a local hematologist, and the diagnosis was made in 2012 through a bone marrow biopsy (Figs. 1 and 2), a BCR/ABL test and a FIP1L1-PDGFRA test; he denied having any complications of the underlying disease until then. During the clinical examination, jugular swelling, bilateral hepatojugular reflux, diffuse crepitations on pulmonary auscultation, systolic murmur in the mitral valve area on cardiac auscultation and edema of the lower limbs were observed.

A chest X-ray performed on admission revealed signs of pulmonary congestion and pleural effusion on the right side; the laboratory analysis revealed normal renal function, a normal electrolyte profile (sodium, potassium, magnesium and calcium), hemoglobin $10.3 \mathrm{~g} / \mathrm{dl}$, hematocrit $28.7 \%, 12,610 / \mathrm{ml}$ total leukocytes with $67.9 \%(8,562 / \mathrm{ml})$ eosinophils, D-dimer $825 \mathrm{ng} / \mathrm{ml}$ (normal range, $190-440 \mathrm{ng} / \mathrm{ml}$ ) and brain natriuretic peptide $187 \mathrm{pg} / \mathrm{ml}$ (normal, <100 pg/ml).

After the laboratory and radiological evaluations, the diagnosis of acute decompensated heart failure with unknown etiology was established. An echocardiogram performed in June 2012 did not reveal structural alterations, with a preserved ejection fraction of the left ventricle, and a segmental and global assessment of the left ventricle without contractility alterations. The patient was admitted to the Cardiology Department for treatment, with simultaneous investigation of the cause underlying the clinical decompensation. A computed tomographic angiography of the chest was performed, which showed no evidence of arterial thromboembolism. Abdominal ultrasonography revealed evidence of splenomegaly, and the transthoracic echocardiogram revealed severe mitral insufficiency with valve retraction, with a regurgitant volume of $56 \mathrm{ml}$ per beat and a regurgitant orifice area of $55 \mathrm{~mm}^{2}$ (Fig. 3).

Daily examinations were performed during hospitalization, which revealed eosinophil levels between 8,562 and $20,150 / \mathrm{ml}$, with variations on a daily basis without changing the dosage of hydroxyurea. The patient was discharged after 9 days of hospitalization with optimization of the clinical treatment and without symptoms.

At $\sim 7$ days after discharge, the patient visited the Emergency Department of HUST with signs of decompensated heart failure, and was again admitted to the Cardiology Department. The patient was reevaluated by an assistant hematologist during the second hospitalization, and the previously performed cytogenetic bone marrow examination showing negativity for BCR/ABL and FIP1L1-PDGFRA was reviewed. After reviewing the treatments performed until that time, treatment with imatinib was recommended due to the rapid cardiac and splenic involvement. Within $24 \mathrm{~h}$ of imatinib therapy, changes in the laboratory tests were apparent, with an eosinophil count of $1,789 / \mathrm{ml}$. By the second day, the eosinophil count was $184 / \mathrm{ml}$. The patient was discharged after 5 days of hospitalization following the significant response to imatinib and compensation of the cardiac symptoms. 

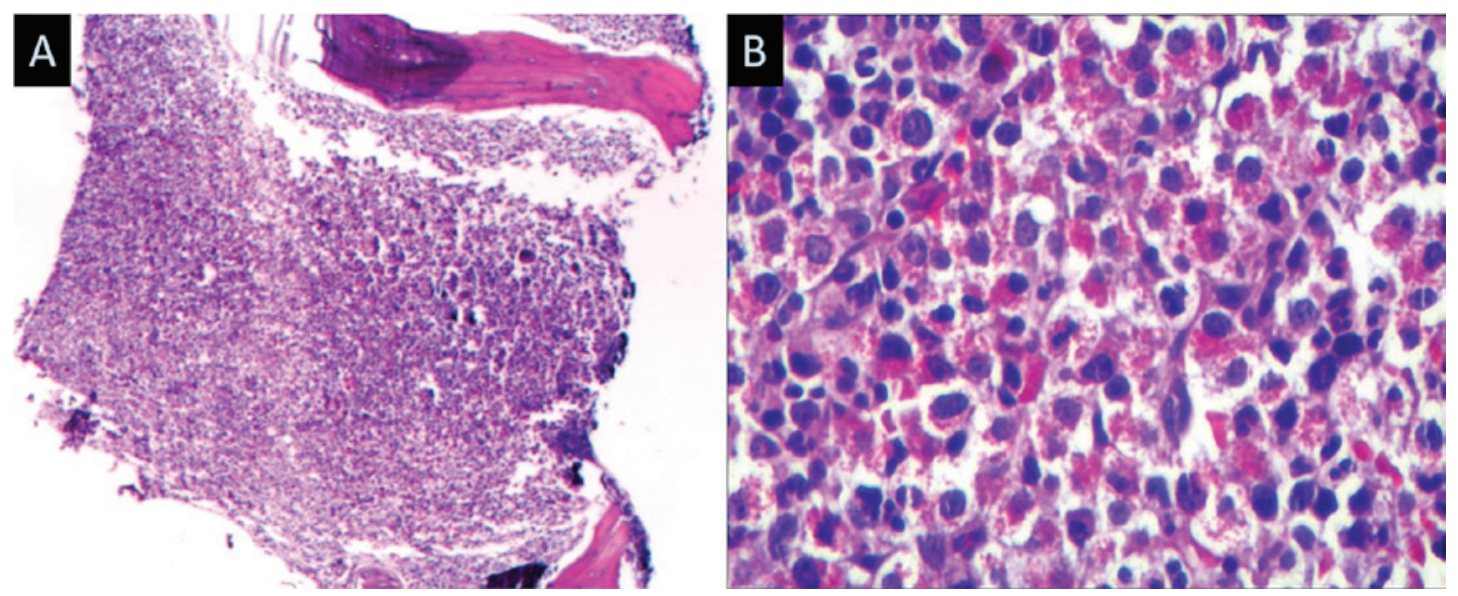

Figure 1. Bone marrow hematoxylin and eosin staining. (A) Magnification, x40: Hypercellular bone marrow with an increased eosinophil count. (B) Magnification, $\mathrm{x} 400$ : Image demonstrating the predominance of eosinophils and eosinophilic precursors.

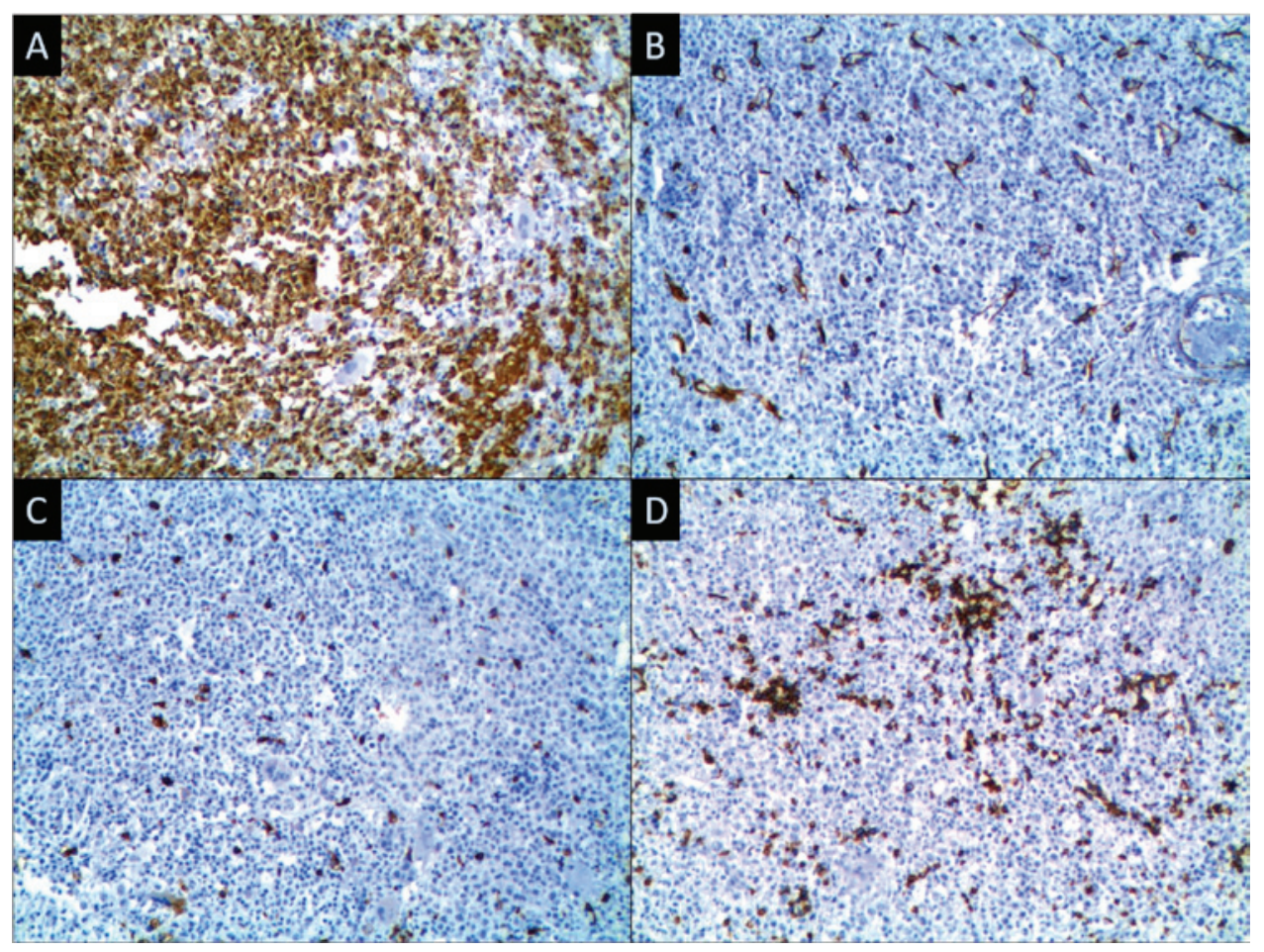

Figure 2. Bone marrow immunohistochemical examination revealed eosinophilic cells (A) positive for myeloperoxidase and negative for (B) CD34, (C) CD117/ c-kit and (D) glycophorin A. Magnification, x100.

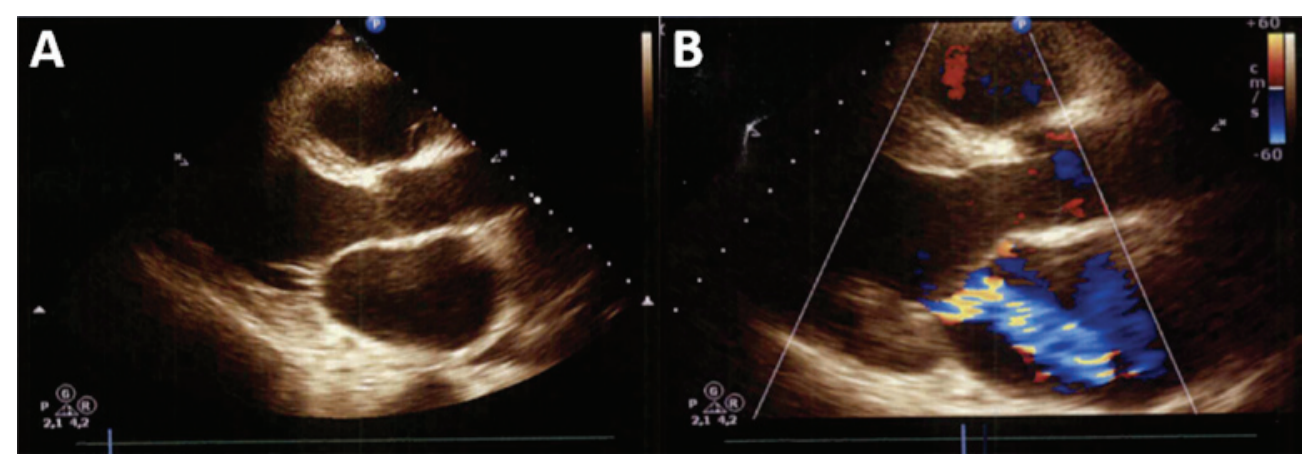

Figure 3. Severe mitral regurgitation with a regurgitant orifice area of $55 \mathrm{~mm}^{2}$, as demonstrated by (A) a two-dimensional transthoracic echocardiogram with (B) color Doppler overlay. 
After 2 weeks, the patient returned to the hospital with an exacerbation of heart failure, with the signs and symptoms of systemic congestion despite the optimization of treatment with oral diuretics. Tests performed on admission revealed the stabilization of the eosinophil count at $21 / \mathrm{ml}$. An optimization of clinical treatment was performed, and subsequent to hemodynamic stabilization, the patient was referred to the cardiac surgery department for replacement of the mitral valve.

The patient returned for follow-up at 8 months after surgery and 9 months from the onset of therapy with imatinib. The patient displayed no cardiovascular symptoms and eosinophil levels were maintained at $40 / \mathrm{ml}$. On the last visit in August 2017, after 19 months of imatinib therapy, the patient presented as stable at HUST, without megaly or adenopathy, using $100 \mathrm{mg} /$ day imatinib, with a hemoglobin value of 13.4, hematocrit of 41.8, 6,352 leucocytes/ml; ( $0 \%$ banded neutrophils, $67.2 \%$ segmented neutrophils, $0.6 \%$ basophils, $0.8 \%$ eosinophils, $10.6 \%$ lymphocytes and $20.8 \%$ monocytes) and 103,700 platelets $/ \mathrm{ml}$.

\section{Discussion}

Hypereosinophilic syndrome is a rare disorder with an unknown incidence rate, which was estimated to be 0.035 per 100,000 individuals in the United States (12). The diagnosis of idiopathic hypereosinophilic syndrome is made following the exclusion of other factors that may lead to increased eosinophil count $(1-3,5)$. In 2008, the World Health Organization proposed a classification based on the subtypes of hypereosinophilia causes, e.g., neoplastic causes and FIP1L1-PDGFRA abnormalities (13). In non-neoplastic cases and following the exclusion of secondary causes, patients are tested for familial hypereosinophilia; if this is excluded, the diagnosis of idiopathic hypereosinophilic syndrome is confirmed (5). In the present case study, a patient with hypereosinophilic syndrome was examined via bone marrow biopsy and BCR/ABL and FIP1L1-PDGFRA status evaluations twice (2012 and 2015), and was found to be negative in both examinations.

The treatment of patients who test negative for FIP1L1-PDGFRA is based on the use of corticosteroids as a first line of therapy (14), with treatment success in $81-85 \%$ of the cases (2), including the improvement of eosinophilia and symptom relief (14). The second line of treatment typically includes hydroxyurea (2); its combination with corticosteroids is recommended, but, despite the reduction in eosinophilia, there is no proven benefit against the natural progression of the disease (15), whereas severe side effects may lead to treatment discontinuation (14). The patient reported use of both therapies for the treatment of his condition; however, despite the partial reduction in the eosinophil count, the disease progressed, with significant cardiac dysfunction and limitations of daily activities.

Treatment with imatinib would be the first therapeutic option for patients testing positive for the FIP1L1-PDGFRA fusion (15); however, previous case studies have demonstrated benefits in FIP1L1-PDGFRA-negative patients as well. In 2007, a study included 72 patients, of whom 63 received imatinib at doses of 100-400 mg per day. Of those patients,
36 were negative for FIP1L1-PDGFRA. A complete response was observed in 4 FIP1L1-PDGFRA-negative patients after 1 month of treatment, and in 1 negative patient after 3 months, totaling an initial response rate of $14 \%$, but with a loss of response after 1-15 months of treatment (16). Among 15 patients who tested negative for FIP1L1-PDGFRA, 6 (40\%) experienced disease relapse after 4-8 months of imatinib treatment at a dose of $400 \mathrm{mg}$ per day (17). In another study with 188 patients, $68(36 \%)$ of whom received imatinib at the maximum dose of $400 \mathrm{mg}$ per day, 43 patients tested negative for FIP1L1-PDGFRA, of whom only 10 (23\%) exhibited a complete $(n=6)$ or partial $(n=4)$ response. By contrast, 88\% (15) of FIP1L1-PDGFRA-positive patients exhibited a good response to treatment (14). Another study divided patients with FIP1L1-PDGFRA into two classes according to the presence of $>4$ criteria suggestive of myeloid neoplasms. In that study, 16 patients negative for FIP1L1-PDGFRA without any criteria of myeloid neoplasms received imatinib, and none of those patients exhibited a clinical or laboratory response to treatment (18).

In the case described herein, the patient had a complete response with $100 \mathrm{mg}$ imatinib after $<48 \mathrm{~h}$ of treatment, with clinical improvement, suggesting that imatinib may achieve good results in certain cases of FIP1L1-PDGFRA fusion-negative hypereosinophilic syndrome. It also suggests a cardiotoxic effect of imatinib, which may have been the triggering factor for the clinical decompensation, despite the progressive laboratorial improvement.

This syndrome may be responsible for the dysfunction of numerous organs, including cardiac involvement in up to $60 \%$ of the cases $(6,10)$. Typically, cardiac involvement in hypereosinophilic syndrome preferentially affects the endocardium and goes through three stages: i) Acute necrosis (initial stage), which is usually asymptomatic and is associated with the infiltration of the myocardium by eosinophils; ii) thrombotic stage, which manifests by progressive damage to the endocardium and may be associated with thrombotic events; and finally, iii) the fibrosis stage, in which the condition progresses to restrictive cardiomyopathy and severe fibrosis, which may cause valvular regurgitation $(2,10,19)$.

In the case presented herein, there was an exacerbation of the heart failure symptoms with evidence of isolated mitral regurgitation, without evidence of associated restrictive cardiomyopathy or left ventricular hypertrophy. Such a clinical presentation is uncommon, as the symptoms of heart failure usually appear during the third stage of disease progression. Two cases of mitral valve involvement in the thrombotic stage were previously described, likely induced by thrombus formation in the endothelium, causing valvular damage $(20,21)$.

In conclusion, according to gathered references, the cardiac involvement in the present case is uncommon, as the clinical changes suggest that the patient had concomitant myocardial dysfunction and fibrosis; however, for unknown reasons, isolated mitral valve involvement was the initial form of cardiac involvement in our patient, which has never been previously described in the literature to the best of our knowledge. The patient in question exhibited disease progression with cardiac and splenic involvement, despite receiving the standard therapy recommended for FIP1L1-PDGFRA-negative patients 
and stable results in the laboratory tests. Due to the limited treatment options, the use of imatinib was selected despite the absence of the FIP1L1-PDGFRA fusion mutation, with a remarkable response at $24 \mathrm{~h}$ after treatment initiation, and maintenance of the low eosinophil levels during the outpatient treatment. However, more studies are required and additional novel treatment options should be tested to further improve the quality of life of patients suffering from hypereosinophilic syndrome.

\section{Acknowledgements}

The authors would like to thank the staff of the Hospital Universitário Santa Terezinha.

\section{Availability of data and materials}

The datasets generated during and/or analyzed during the current study are available from the corresponding author on reasonable request.

\section{Funding}

No funding was received.

\section{Ethics approval and consent to participate}

The present study was approved by the Institutional Ethics Committee (Comitê de Ética em Pesquisa Unoesc/HUST; approval no. 160968).

\section{Consent for publication}

Consent for publication was obtained from the patient.

\section{Authors' contributions}

ASDB: Study concept and design; acquisition of data; analysis and interpretation of data; drafting of the manuscript; critical revision of the manuscript. RC: Analysis and interpretation of data; drafting of the manuscript; critical revision of the manuscript. ESM: Analysis and interpretation of data; drafting of the manuscript; critical revision of the manuscript. ARB: Study concept and design; analysis and interpretation of data; drafting of the manuscript; critical revision of the manuscript.

\section{Competing interests}

The authors declare that they have no competing interests.

\section{References}

1. Roufosse F: Management of hypereosinophilic syndromes. Immunol Allergy Clin N Am 35: 561-575, 2015.

2. Cools J, DeAngelo DJ, Gotlib J, Stover EH, Legare RD, Cortes J, Kutok J, Clark J, Galinsky I, Griffin JD, et al: A tyrosine kinase created by fusion of the PDGFRA and FIP1L1 genes as a therapeutic target of imatinib in idiopathic hypereosinophilic syndrome. N Engl J Med 348: 1201-1214, 2003.

3. Klion AD: How I treat hypereosinophilic syndromes. Blood 126 1069-1077, 2015
4. Hardy WR and Anderson RE: The hypereosinophilic syndromes. Ann Intern Med 68: 1220-1229, 1968.

5. Mankad R, Bonnichsen C and Mankad S: Hypereosinophilic syndrome: Cardiac diagnosis and management. Heart 102: 100-106, 2016.

6. Cogan E and Roufosse F: Clinical management of the hypereosinophilic syndromes. Expert Rev Hematol 5: 275-289, 2012.

7. Ronchi Junior I, Vasconcelos Krebs CN, Pietrovicz J, Nocera VB, Pedri LE, Fouani MM, Lopes GL, Loidi de Santana WM, Akiyoshi C and Henriques C: Síndrome hipereosinofílica idiopática. Relato de caso e revisão de literatura. Rev Bras Clin Med 8: 177-182, 2010.

8. Kalac M, Quintás-Cardama A, Vrhovac R, Kantarjian H and Verstovsek S: A critical appraisal of conventional and investigational drug therapy in patients with hypereosinophilic syndrome and clonal eosinophilia. Cancer 110: 955-964, 2007.

9. Brito-Babapulle F: The eosinophilias, including the idiopathic hypereosinophilic syndrome. Br J Haematol 121: 203-223, 2003.

10. Benezet-Mazuecos J, Marcos-Alberca P, Farré J, Orejas M, de la Fuente A and Prieto E: Images in cardiovascular medicine. Early differential resolution of right and left ventricular obliteration in Löffler endocarditis after chemotherapy and anticoagulation. Circulation 114: e635-e637, 2006.

11. Curtis $\mathrm{C}$ and Ogbogu P: Hypereosinophilic syndrome. Clinic Rev Allerg Immunol 50: 240-251, 2016.

12. Crane MM, Chang CM, Kobayashi MG and Weller PF: Incidence of myeloproliferative hypereosinophilic syndrome in the United States and an estimate of all hypereosinophilic syndrome incidence. J Allergy Clin Immunol 126: 179-181, 2010.

13. Bain BJ, Gilliland DG, Horny HP, et al: Myeloid and lymphoid neoplasms with eosinophilia and abnormalities of PDGFRA, PDGFRB or FGFR1. World Health Organization classification of tumors of hematopoietic and lymphoid tissues: 68-73, Lyon. France: IARC Press, 2008.

14. Ogbogu PU, Bochner BS, Butterfield JH, Gleich GJ, Huss-Marp J, Kahn JE, Leiferman KM, Nutman TB, Pfab F, Ring J, et al: Hypereosinophilic syndrome: A multicenter, retrospective analysis of clinical characteristics and response to therapy. J Allergy Clin Immunol 124: 1319-1325.e3, 2009.

15. Gotlib J: World Health Organization-defined eosinophilic disorders: 2015 update on diagnosis, risk stratification, and management. Am J Hematol 90: 1078-1089, 2015.

16. Baccarani M, Cilloni D, Rondoni M, Ottaviani E, Messa F, Merante S, Tiribelli M, Buccisano F, Testoni N, Gottardi E, et al: The efficacy of imatinib mesylate in patients with FIP1L1-PDGFRalpha-positive hypereosinophilic syndrome. Results of a multicenter prospective study. Haematologica 92: 1173-1179, 2007.

17. Metzgeroth G, Walz C, Erben P, Popp H, Schmitt-Graeff A, Haferlach C, Fabarius A, Schnittger S, Grimwade D, Cross NC, et al: Safety and efficacy of imatinib in chronic eosinophilic leukaemia and hypereosinophilic syndrome: A phase-II study. Br J Haematol 143: 707-715, 2008.

18. Khoury P, Desmond R, Pabon A, Holland-Thomas N, Ware JM, Arthur DC, Kurlander R, Fay MP, Maric I and Klion AD: Clinical features predict responsiveness to imatinib in platelet-derived growth factor receptor-alpha-negative hypereosinophilic syndrome. Allergy 71: 803-810, 2016.

19. Weller PF and Bubley GJ: The idiopathic hypereosinophilic syndrome. Blood 83: 2759-2779, 1994.

20. Madhwal S, Goldberg J, Barcena J, Guha A, Gogate P, Cmolik B and Elgudin Y: Unusual cause of acute mitral regurgitation: Idiopathic hypereosinophilic syndrome. Ann Thorac Surg 93: 974-977, 2012.

21. Kim NK, Kim CY, Kim JH, Jang SY, Bae MH, Lee JH, Yang DH, Park HS, Cho Y and Chae SC: A hypereosinophilic syndrome with cardiac involvement from thrombotic stage to fibrotic stage. J Cardiovasc Ultrasound 23: 100-102, 2015. 